Relations industrielles

Industrial Relations

\title{
Labor Relations in the Lithographic Industry, Fred C. Munson, Harvard University Press. 1963. 272 pages.
}

\section{Mathieu Vaillancourt}

Volume 21, numéro 2, 1966

URI : https://id.erudit.org/iderudit/027689ar

DOI : https://doi.org/10.7202/027689ar

Aller au sommaire du numéro

Éditeur(s)

Département des relations industrielles de l'Université Laval

ISSN

0034-379X (imprimé)

1703-8138 (numérique)

Découvrir la revue

Citer ce compte rendu

Vaillancourt, M. (1966). Compte rendu de [Labor Relations in the Lithographic Industry, Fred C. Munson, Harvard University Press. 1963. 272 pages.] Relations industrielles / Industrial Relations, 21(2), 304-305.

https://doi.org/10.7202/027689ar

Tous droits réservés @ Département des relations industrielles de l'Université Laval, 1966
Ce document est protégé par la loi sur le droit d'auteur. L’utilisation des services d'Érudit (y compris la reproduction) est assujettie à sa politique d'utilisation que vous pouvez consulter en ligne.

https://apropos.erudit.org/fr/usagers/politique-dutilisation/ 
ployées pour l'échantillonnage de mesures et d'analyses des poussières en suspension dans I'air. Tout ceci se fait au moyen d'apporeils, appareils dont on nous explique la nature, le fonctionnement et l'utilité à mesure que progresse leur énumération.

Un bref exposé destiné à faire voir les avantages et la nécessité de former les cadres, les travailleurs et les agents de sécurité vient compléter cette étude qui peut être qualifiée de complète, précise et très pratique.

\section{Ronald Pleau}

Pareto \& Mosca, Edited by James H. Meisel, Series The Makers of Modern Social Science, Prentice Hall, Inc., Englewood Cliffs, N.J., 1965. 192 poges.

Vilfredo Pareto (1848-1923) et Gaetano Mosca (1858-1941) demeurent des personnages associés dans l'histoire de la pensée occidentale. Les deux hommes rejetèrent l'idéal d'égalitarisme en démocratie aussi bien que le déterminisme économique de Marx. Tous deux formulèrent aussi, de différentes façons, les théories traditionnelles sur le pouvoir politique.

Ainsi ce volume présente des explications et critiques sur ces deux théoristes. Quelques-uns des penseurs sociaux et politiques les plus connus, tel Talcott Parson, Raymond Aaron, Wright Mills, etc., révèlent I'inconsistance, les faussetés ou le réalisme des idées de Pareto et Mosca.

Les deux auteurs furent les pionniers d'une nouvelle approche des sciences sociales. Mosca a développé * a model of historical power structures which transcended and denied the traditional Aristotelian classification of governments (p. 2). Pareto chercha « a system through which to predict the laws of change as well as of stability \# (p. 2).

De plus, même si Pareto et Mosca ont insisté sur le fossé entre ceux qui contrôlent le pouvoir et les gouvernés, leur vue sur l'irrationalité de l'homme et leur croyance que les règles politiques doivent reposer sur l'emploi du mythe et de la force, donna peu d'armes aux forces d'extrême droite.

Enfin, comme le soulignent les signataires de ces articles, les deux théoriciens italiens ont influencé por les questions qu'ils ont abordées, la pensée sociologique libérale.

Soulignons certains articles:

- Le système sociologique de Poreto
- La morphologie et la psycholagie sociale de Pareto

- La politique «paretienne

- Mosca et les leçons politiques de l'histoire

- L'élite politique et la bureaucratie (Mosco).

\section{Fernand Blais}

Labor Relations in the Lithographic Industry, Fred C. Munson, Harvard University Press. 1963. 272 pages.

Ce livre débute en rappelant brièvement la notion de système de relations industrielles avancée par John T. Dunlop, quels en sont les acteurs et comment les trois facteurs suivants i.e. la technologie, les pressions du marché et la distribution du pouvoir dans la société, influencent la naissance et l'évolution des règles du système.

Après avoir défini ce qu'est l'industrie lithographique, on aborde la situation des acteurs-clés du système: les employeurs, leurs organisations, la structure des unités patronales de négociation ainsi que les syndicats dans l'industrie concernée et leurs structures. Considérant ensuite le contexte technologique l'auteur, après avoir décrit le procédé lithographique, commente l'influence des innovations techniques sur l'organisation de l'industrie. Selon Munson, ces innovations ont eu les effets suivants: le Rapide accroissement de l'importance relative de la petite firme; $2 e$-Elimination de certaines tôches spécialisées et création de nouvelles; $3 e-$ Extension du procédé à d'autres industries; $4 \mathrm{e}$ - Disparition des différences entre les divers procédés dans I'imprimerie; $5 e$-Acceptation par la ALA (Amalgamated Lithographers of America) de ces changements technologiques.

Par ailleurs, les règles d'un système de relations industrielles subissent aussi $I^{\prime}$ influence de caractéristiques économiques, telles les variations dans le produit, les mécanismes du marché et l'emplacernent des firmes par rapport aux marchés. Dans I'industrie lithographique, I'expansion du marché a surtout eu pour effet d'affaiblir I'unité patronale à la table de négociation, en diversifiant la production et de raffermir la position des syndicats de moindre importance.

Ayant ensuite établi la valeur de la force de travail dans cette industrie, Fred C. Munson présente d'abord la situation qui prévalait avant les années trente dans le système, puis celle qui suivit jusqu'en 1945, 
pour enfin peindre le système qui suivit la deuxième grande guerre.

Dans un système de relations industrielles comme celui qui prévaut dans I'industrie lithographique, la négociation des contrats collectifs de travail semble être affectée par l'individualisme des employeurs et des syndicats locaux, malgré l'effort pour négocier à l'échelle nationale. $\mathrm{Ce}$ niveau amène donc assez souvent des négociations informelles exigées par le climat de désaccord qui peut exister au moment du renouvellement d'une convention.

Depuis 1945, les travailleurs de l'industrie lithographique ont obtenu des conditions plus que favorables: semaine de travail de 35 heures pour la majorité d'entre eux, plans d'assurance-santé, d'assurance-vie et de pension. De plus, il semble qu'ils aient progressé plus ropidement sur le terrain des salaires que la plupart des travailleurs dans les autres métiers de l'imprimerie. Et comme conséquence de la négociation dans cette industrie, on a vu apparaittre un contrôle de l'offre de travail par les syndicats, contrôle qui s'est concrétisé d'une part par des restrictions sur le nombre de travailleurs et d'autre part par des restrictions sur les heures de travail. Le premier type de contrôle a donc amené une restriction de I'offre alors que le second servait d̀ créer une demande additionnelle. L'auteur termine son exposé en résumant la situation actuelle et en esquissant les tendances qui se dessinent pour l'avenir.

\section{Mathieu Vaillancourt}

The Management of Personnel Relations, History and Origins, Cyril C. Ling, Richard D. Irwin, Inc., Homewood, Illinois. 1965. 554 pages.

Comme l'indique bien le titre de ce volume, nous retrouvons dans ses pages I'histoire de la croissance de la fonction du personnel dans les firmes américaines.

Cet ouvrage volumineux est divisé en cinq grandes parties. Dans une première l'auteur introduit son sujet de façon à expliquer les différentes questions dont il traitera par la suite.

II débute son exposé par une description de l'évolution du contexte économique et social dans lequel a évolué la fonction du personnel après quoi il nous fournit un cadre de référence destiné à faciliter la définition et la compréhension des concepts employés dans cette étude. Puis il poursuit en nous donnant quelques origines de la pensée concernant le personnel pour terminer par une explication du plan de son volume avec les limitations et les avantages d'un tel travail.

Dans l'élaboration des faits historiques qui constituent la matière des quatre parties subséquentes, on remarque que l'ordre chronologique a été suivi. Ainsi le contenu se divise selon les périodes suivantes: de 1400 à 1890 (Partie II) i.e. le premier stage de développement, de 1890-1920 (Partie III) le second stage de développement, de 1920 à nos jours ou troisième stage de développement. Dans la partie $V$, on retrouve un bref sommaire de ce qui $s^{\prime}$ est fait dans le passé suivi d'un essai de projection pour savoir ce qui pourrait survenir dans l'avenir dans le domaine du personnel.

Dans la partie II donc, ce sont les influences de l'évolution industrielle et technologique, du mouvement du "Welfare », du mouvement syndical, de la législation dú travail, de la législation industrielle sur la fonction du personnel et des contributions à l'éducation de cette dernière qui font l'objet d'un examen plus approfondi. Ces sujets constituent autant de chapitres à l'intérieur desquels l'auteur passe en revue l'évolution industrielle de l'Europe et des Etats-Unis avec son impact sur la fonction du personnel (chap. 2), la définition, les objectifs, la patience, le travail dans la guilde et dans I'industrie moderne du mouvement du « Welfare » (chap. 3), les formes d'apprentissage, les débuts de l'éducation dans l'industrie et les facteurs politiques qui l'ont influencée (chap. 4), la croissance, les effets de la croissance des syndicats américains et de la législation ouvrière (chap. 5), l'évolution des lois concernant les salaires et les heures de travail, leurs effets sur la fonction du personnel, les lois de sécurité et d'accident du travail (chap. 6).

La section suivante est consacrée à la modernisation de la médecine industrielle, à ses origines, à son évolution, son statut, ses problèmes en relations avec le personne! (chap. 7), à la psychologie industrielle, ses origines, son développement et les contributions qu'elle a apportées à cette fonction de l'entreprise (chap. 8) aux contributions de la pensée du management i.e. du « planning " ou la prise de décision, à l'organisation, au leadership, à la motivation et au contrôle (chap. 9) à l'influence de la seconde guerre mondiale, et à ses effets sur les relations avec les employés (chap. 10), aux contributions internes telles les tendances vers la professionalisation (chap. 11). 